01

\title{
Электрическая площадь поля в вакууме с движущимися зарядами
}

\author{
(C) Н.Н. Розанов
}

Физико-технический институт им. А.Ф. Иоффе РАН, Санкт-Петербург, Россия

E-mail: nnrosanov@mail.ru

Поступило в Редакцию 5 ноября 2019г.

В окончательной редакции 5 ноября 2019г.

Принято к публикации 8 ноября 2019г.

\begin{abstract}
Представлено точное аналитическое выражение для электрической площади поля, генерируемого движением заряженных частиц с постоянным ускорением. Приведена приближенная форма пространственного распределения электрической площади в окрестности точки мгновенной остановки зарядов. Показана возможность генерации квазиуниполярных импульсов электромагнитного излучения со значительной электрической площадью.
\end{abstract}

Ключевые слова: излучение движущихся зарядов, импульсы электромагнитного излучения.

DOI: 10.21883/PJTF.2020.04.49043.18094

Импульсы электромагнитного поля, у которых имеется постоянная составляющая, включая униполярные импульсы, впервые, по-видимому, были рассмотрены в работах [1-3]. В дальнейшем было показано, что квазиуниполярные импульсы оказывают значительно более сильное воздействие на микрообъекты (как классические заряды [4], так и принципиально квантовые атомы [5-8] и молекулы [9], см. также обзор [10]), чем обычные биполярные. Это вызвано их фактически однонаправленным воздействием на объекты (в отличие от разнонаправленного для биполярных импульсов). Несмотря на сообщения об экспериментальном наблюдении униполярных импульсов излучения [11], возможность их существования и сейчас порой подвергается сомнению. Отмеченное выше подчеркивает актуальность надежной демонстрации такой возможности и поиска схем генерации импульсов со значительной электрической площадью импульса, определяемой соотношением

$$
\mathbf{S}_{E}(\mathbf{r})=\int_{t=-\infty}^{+\infty} \mathbf{E}(\mathbf{r}, t) d t
$$

В этом соотношении $\mathbf{r}$ и $t-$ пространственный радиусвектор и время, а $\mathbf{E}-$ напряженность электрического поля.

Ряд чисто оптических методов генерации квазиуниполярных импульсов обсуждается в обзоре [12]. Однако в [12] и цитированной там литературе рассмотрение проводилось в рамках одномерной геометрии, т.е. в плосковолновом приближении, что ограничивает привязку результатов к реальным схемам. Целью настоящей работы служит демонстрация возможности получения трехмерных квазиуниполярных импульсов в вакууме с ускоренно движущимися зарядами и достижения больших значений электрической площади при генерации излучения в простом варианте такого движения. Для этого используется следующая из точных электродинамических уравнений Максвелла в вакууме с зарядами $[13,14]$ замкнутая система уравнений для электрической площади [15].

Согласно [15], для определенной выше электрической площади $\mathbf{S}_{E}$ справедливы следующие уравнения, совпадающие с точностью до обозначений с уравнениями электростатики:

$$
\operatorname{rot} \mathbf{S}_{E}=0, \quad \operatorname{div} \mathbf{S}_{E}=4 \pi Q .
$$

Здесь $Q(\mathbf{r})=\int_{-\infty}^{+\infty} \rho(\mathbf{r}, t) d t$, где $\rho-$ плотность электрических зарядов. Тем самым $Q(\mathbf{r})$ имеет смысл интегральной плотности зарядов, прошедших окрестность точки $\mathbf{r}$, с учетом времени пребывания в этой окрестности. Величина $Q$ обращается в бесконечность в случае статических зарядов, так что к этому случаю рассмотрение неприменимо.

Система (1) позволяет определить электрическую площадь $\mathbf{S}_{E}$ по заданному распределению интегральной плотности заряда $Q(\mathbf{r})$. Для этого вводится „потенциал“ $\Phi_{S}$, подчиняющийся уравнению Пуассона,

$$
\Delta \Phi_{S}=-4 \pi Q
$$

Решение (2) записывается в виде

$$
\Phi_{S}(\mathbf{r})=\int \frac{Q\left(\mathbf{r}^{\prime}\right)}{\left|\mathbf{r}-\mathbf{r}^{\prime}\right|} d \mathbf{r}^{\prime} .
$$

Наконец, электрическая площадь определяется через градиент ,потенциала“

$$
\mathbf{S}_{E}=-\operatorname{grad} \Phi_{S}
$$

Конкретизируем общее решение простым вариантом ускоренного нерелятивистского движения зарядов. Пусть узкий сгусток не взаимодействующих между собой частиц с общим зарядом $q$ вылетает из электронной пушки с начальной скоростью $\mathbf{v}_{0}=v_{0} \mathbf{e}_{z}$ вертикально вверх $\left(\mathbf{e}_{z}-\right.$ орт вдоль оси $\left.z\right)$. Под действием силы 
тяготения частицы движутся с ускорением $\mathbf{g}=-g \mathbf{e}_{z}$, где $g$ - ускорение свободного падения. Соответственно частицы достигают максимальной высоты $z_{m}$ и затем падают вниз, попадая через время $2 t_{0}$ в пушку и исчезая там.

Решение уравнения движения $d^{2} z / d t^{2}=-g$ с начальными условиями $z=0, v=v_{0}$ при $t=-t_{0}$ приводит к следующим соотношениям:

$$
\begin{gathered}
z=z_{m}-\frac{1}{2} g t^{2}, v_{0}=g t_{0}, \\
z_{m}=\frac{1}{2} g t_{0}^{2}=\frac{v_{0}^{2}}{2 g}, \quad v(z)= \pm \sqrt{2 g\left(z_{m}-z\right)} .
\end{gathered}
$$

Примем теперь дельтообразное распределение плотности заряда

$$
\rho(r, z)=q \delta(r) \delta\left(z-z_{q}(t)\right), \quad r=\sqrt{x^{2}+y^{2}},
$$

где $x$ и $y$ - поперечные координаты. Тогда интегральная плотность заряда

$$
\begin{aligned}
Q(r, z) & =2 \int_{0}^{t_{0}} \rho(r, z, t) d t=\frac{2 q}{|v(z)|} \delta(r) \\
& =\sqrt{\frac{2}{g\left(z_{m}-z\right)}} q \delta(r) .
\end{aligned}
$$

Хотя помимо дельтообразной зависимости от $r$ выражение для $Q$ обладает особенностью при $z=z_{m}$ (вершина траектории, мгновенная скорость обращается в нуль), эта особенность устраняется в выражении для „потенциала“ $\Phi_{S}$. Для него при $z>z_{m}$ находим

$$
\begin{aligned}
\Phi(r, z) & =q \sqrt{\frac{2}{g}} \int_{0}^{z_{m}} \frac{d z^{\prime}}{\sqrt{\left(z_{m}-z^{\prime}\right)\left[r^{2}+\left(z-z^{\prime}\right)^{2}\right]}} \\
& =q \sqrt{\frac{2}{g p}} F\left(2 \operatorname{arcctg} \sqrt{\frac{z_{m}}{p}}, \sqrt{\frac{p-z+z_{m}}{2 p}}\right) .
\end{aligned}
$$

Здесь $p=\sqrt{r^{2}+\left(z_{m}-z\right)^{2}}, F-$ эллиптический интеграл первого рода [16].

Вид ,потенциала“ резко упрощается при $r=0$ :

$$
\Phi(0, z)=-2 q \sqrt{\frac{2}{g\left(z-z_{m}\right)}} \operatorname{arctg} \sqrt{\frac{z_{m}}{z-z_{m}}} .
$$

Учтем теперь осевую симметрию „потенциала“ (соответственно азимутальная составляющая градиента „потенциала“ обращается в нуль) и то, что разложение $\Phi$ по $r$ содержит четные степени $r$ (из-за этого вблизи оси (малые $r$ ) радиальная составляющая градиента пропорциональна $r$ и потому мала). Поэтому при малых $r$ из
(9) следует

$$
\begin{gathered}
\mathbf{S}_{E} \approx \mathbf{e}_{z} q \sqrt{\frac{2}{g}\left(z-z_{m}\right)^{-1 / 2}\left\{\frac{1}{z-z_{m}}\right.} \\
\left.\operatorname{arctg} \sqrt{\frac{z_{m}}{z-z_{m}}}-\frac{1}{2 z}\right\} .
\end{gathered}
$$

При $z-z_{m} \ll z_{m}$

$$
\mathbf{S}_{E} \approx \mathbf{e}_{z} q \pi \sqrt{\frac{1}{2 g}}\left(z-z_{m}\right)^{-3 / 2} .
$$

Видно, что электрическая площадь направлена вдоль оси $z$ и неограниченно возрастает при приближении к $z_{m}$. Если микрообъект (например, атом) все время движения зарядов находился в окрестности $z_{m}$, то на него будет воздействовать однонаправленный квазиуниполярный импульс с весьма большой электрической площадью. Ее величину можно увеличивать до предела, ограниченного точностью фиксации координат микрообъекта и размытостью электронного сгустка.

\section{Благодарности}

Автор благодарен М.В. Архипову за полезные обсуждения.

\section{Конфликт интересов}

Автор заявляет, что у него нет конфликта интересов.

\section{Список литературы}

[1] Бессонов Е.Г. // ЖЭТФ. 1981. Т. 80. В. 3. С. 852-858.

[2] Bessonov E.G. // Nucl. Instr. Meth. A. 1991. V. 308. P. 135 139.

[3] Бессонов Е.Г. // Квантовая электроника. 1992. Т. 19. № 1. C. 35-39.

[4] Розанов Н.Н., Высотина Н.В. // ЖЭТФ. 2019. Т. 156. B. 6 (12). C. 1-4.

[5] Dimitrovski D., Solov'ev E.A., Briggs J.S. // Phys. Rev. Lett. 2004. V. 93. P. 083003.

[6] Dimitrovski D., Solov'ev E.A., Briggs J.S. // Phys. Rev. A. 2005. V. 72. P. 043411.

[7] Макаров Д.Н., Матвеев В.И. // Письма в ЖЭТФ. 2016. T. 103. B. 6. C. $464-468$.

[8] Розанов Н.Н. // Оптика и спектроскопия. 2018. Т. 124. В. 1. C. $75-77$.

[9] Arkhipov R.M., Arkhipov M.V., Babushkin I.V., Demircan A., Morgner U., Rosanov N.N. // Opt. Lett. 2019. V. 44. P. 1202 1205.

[10] Розанов Н.Н., Архипов Р.М., Архипов М.В. // УФН. 2018. T. 188. № 12. C. 1347-1353.

[11] Naumenko G., Shevelev M.V. // J. Instrum. 2018. V. 13. P. C05001.

[12] Архипов Р.М., Архипов М.В., Шимко А.А., Пахомов А.В., Розанов Н.Н. // Письма в ЖЭТФ. 2019. Т. 109. В. 10. С. 920. 
[13] Ландау Л.Д., Лифиии, Е.М. Теория поля. М.: Физматлит, 1973. $400 \mathrm{c}$

[14] Ландау Л.Д., Лифиии, Е.М. Электродинамика сплошных сред. М.: Физматлит, 1982.620 с.

[15] Розанов Н.Н. // Оптика и спектроскопия. 2020. Т. 128. В. 1. C. 95-97.

[16] Градштейн И.С., Рыжик И.М. Таблицы интегралов, сумм, рядов и произведений. М.: Физматлит, 1962.1100 с. 